HEP/123-qed

\title{
Improved Nucleon Properties in the Extended Quark Sigma Model
}

\author{
M. Abu-Shady \\ Department of Mathematics, Faculty of Science, Menoufia University, Egypt
}

(Date textdate; Received textdate; Revised textdate; Accepted textdate; Published textdate)

\begin{abstract}
The quark sigma model describes the quarks interacting via exchange the pions and sigma meson fields. A new version of mesonic potential is suggested in the frame of some aspects of the quantum chromodynamics (QCD). The field equations have been solved in the mean-field approximation for the hedgehog baryon state. The obtained results are compared with previous works and other models. We conclude that the suggested mesonic potential successfully calculates nucleon properties..
\end{abstract}




\section{Contents}

I. Introduction

II. The Chiral-Quark Sigma Model

III. The Chiral Higher-Order Quark Sigma Model

IV. Numerical Calculations and Discussions

A. The scalar field $\sigma^{\prime}$

106

B. The pion field $\pi$

107

C. The Properties of the Nucleon

108

D. Discussion of the Results

\section{Comparison with Other Models}

\section{Conclusion}

VII. References

\section{Introduction}

The description of the processes involving strong interactions is very difficult in the frame of the quantum chromodynamics (QCD) due to its non-abelian color and flavor structure and strong coupling constants. These effective models, like quark sigma model, which are constructed in such a way as to respect general properties from the more fundamental theory (QCD), such as the chiral symmetry and its spontaneous breaking [1]. It is known that the linear sigma model of Gell-Mann and Levy [2] does not always give the correct phenomenology such as the value of the isoscalar pion-nucleon scattering length is too large as in Refs. [3-5]. Birse and Banerjee [3] constructed equations of motion treating both $\sigma$ and $\pi$ fields as time-independence classical fields and the quarks in hedgehog spinor state. This work is reexamined by Broniowski and Banerjee [4] with corrected numerical errors in Ref. [3]. Birse [5] generalized this mean-field approximation to include angular momentum and isospin projection.

Recently, the mesons play an important role for improving the nucleon properties in 
the chiral quark models. In the framework of the perturbative chiral quark model $[6,7]$ which extended to include the kaon and eta mesons cloud contributions to analyze the electromagnetic structure of nucleon. Horvat et al. [8] applied Tamm-Dancoff method to the chiral quark model which extended to include additional degrees of freedom as a pseudoscalar isoscalar field and a triplet of scalar isovector to provide a better description of nucleon properties. In Refs. [9-11], the authors analyzed a particular extension of the linear sigma model coupled to valence quarks in which contained an additional term with gradients of the chiral fields and investigated the dynamically consequence of this term and its relevant to the phenomenology. In addition, Rashdan et al. [12, 13] 1and Abu-shady [14] increased the order mesonic interactions in the chiral quark sigma model using mean-field approximation to improve nucleon properties.

The aim of the paper is to introduce the suggested mesonic potential to improve nucleon properties and avoid the difficulty which found in the previous works. The paper is organized as follow: In the following Section, we review briefly the linear sigma model. The higherorder mesonic interactions are studied in details in Sec. 3. The numerical calculations and the discussion of results are presented in Secs. 4 and 5, respectively.

\section{THE CHIRAL-QUARK SIGMA MODEL}

Brise and Banerjee [3] described the interactions of quarks via the exchange of $\sigma$ and $\pi$ - meson fields. The Lagrangian density is

$$
L(r)=i \bar{\Psi} \partial_{\mu} \gamma^{\mu} \Psi+\frac{1}{2}\left(\partial_{\mu} \sigma \partial^{\mu} \sigma+\partial_{\mu} \pi . \partial^{\mu} \pi\right)+g \bar{\Psi}\left(\sigma+i \gamma_{5} \tau . \pi\right) \Psi-U_{1}(\sigma, \pi)
$$

with

$$
U_{1}(\sigma, \pi)=\frac{\lambda^{2}}{4}\left(\sigma^{2}+\pi^{2}-\nu^{2}\right)^{2}+m_{\pi}^{2} f_{\pi} \sigma,
$$

is the meson-meson interaction potential where the $\Psi, \sigma$ and $\pi$ are the quark, sigma, and pion fields, respectively. In the mean-field approximation, the meson fields treat as timeindependent classical fields. This means that we replace the power and the products of the meson fields by the corresponding powers and the products of their expectation values. In Eq. (2), the meson-meson interactions leads to the hidden chiral symmetry $S U(2) \times S U(2)$ with $\sigma(r)$ taking on a vacuum expectation value

$$
\langle\sigma\rangle=-f_{\pi},
$$


where $f_{\pi}=92.4 \mathrm{MeV}$ is the pion decay constant. The final term in Eq. (2) is included to break the chiral symmetry explicitly. It leads to the partial conservation of axial-vector current (PCAC). The parameters $\lambda^{2}, \nu^{2}$ can be expressed in terms of $f_{\pi}$ and the masses of mesons as,

$$
\begin{aligned}
\lambda^{2} & =\frac{m_{\sigma}^{2}-m_{\pi}^{2}}{2 f_{\pi}^{2}}, \\
\nu^{2} & =f_{\pi}^{2}-\frac{m_{\pi}^{2}}{\lambda^{2}} .
\end{aligned}
$$

\section{THE CHIRAL HIGHER-ORDER QUARK SIGMA MODEL}

The Lagrangian density of the extended linear sigma model which describes the interactions between quarks via the $\sigma$ and $\pi$ mesons [14]

$$
L(r)=i \bar{\Psi} \gamma_{\mu} \partial^{\mu} \Psi+\frac{1}{2}\left(\partial_{\mu} \sigma \partial^{\mu} \sigma+\partial_{\mu} \pi . \partial^{\mu} \pi\right)+g \bar{\Psi}\left(\sigma+i \gamma_{5} \tau . \pi\right) \Psi-U_{2}(\sigma, \pi)
$$

with

$$
\begin{aligned}
U_{2}(\sigma, \pi) & =\frac{\lambda_{1}^{2}}{4}\left(\sigma^{2}+\pi^{2}-\nu_{1}^{2}\right)^{2}+\frac{\lambda_{2}^{2}}{4}\left(\left(\sigma^{2}+\pi^{2}\right)^{2}-\nu_{2}^{2}\right)^{2} \\
& +m_{\pi}^{2} f_{\pi} \sigma .
\end{aligned}
$$

It is clear that potential satisfies the chiral symmetry when $m_{\pi} \rightarrow 0$. In the original model [3], the higher-order term in Eq. 7 is excluded by the requirement of renormalizability. Since

we are going to use Eq. (7) as an approximating effective model. The model did not need and should not be renormalizable as in Ref. [9]. By using the PCAC and the minimization conditions of mesonic potential [14], we obtain

$$
\begin{aligned}
& \lambda_{1}^{2}=\frac{m_{\sigma}^{2}-m_{\pi}^{2}}{4 f_{\pi}^{2}}, \quad \nu_{1}^{2}=f_{\pi}^{2}-\frac{m_{\pi}^{2}}{\lambda_{1}^{2}}, \\
& \lambda_{2}^{2}=\frac{m_{\sigma}^{2}-3 m_{\pi}^{2}}{16 f_{\pi}^{6}}, \quad \nu_{2}^{2}=f_{\pi}^{4}-\frac{m_{\pi}^{2}}{2 \lambda_{2}^{2} f_{\pi}^{2}} .
\end{aligned}
$$

Now we can expand the extremum with the shifted field defined as

$$
\sigma=\sigma^{\prime}-f_{\pi}
$$

substituting Eq. (10) into Eq. (6), we get 


$$
\begin{aligned}
L(r) & =i \bar{\Psi} \gamma_{\mu} \partial^{\mu} \Psi+\frac{1}{2}\left(\partial_{\mu} \sigma^{\prime} \partial^{\mu} \sigma^{\prime}+\partial_{\mu} \pi . \partial^{\mu} \pi\right)-g \bar{\Psi} f_{\pi} \Psi+g \bar{\Psi} \sigma^{\prime} \Psi+i g \bar{\Psi} \gamma_{5} . \pi \Psi \\
& -U_{2}\left(\sigma^{\prime}, \pi\right)
\end{aligned}
$$

with

$$
\begin{aligned}
U_{2}\left(\sigma^{\prime}, \pi\right) & =\frac{\lambda_{1}^{2}}{4}\left(\left(\sigma^{\prime}-f_{\pi}\right)^{2}+\pi^{2}-\nu_{1}^{2}\right)^{2}+\frac{\lambda_{2}^{2}}{4}\left(\left(\left(\sigma^{\prime}-f_{\pi}\right)^{2}+\pi^{2}\right)^{2}-\nu_{2}^{2}\right)^{2} \\
& +m_{\pi}^{2} f_{\pi}\left(\sigma^{\prime}-f_{\pi}\right) .
\end{aligned}
$$

The time-independent fields $\sigma^{\prime}(r)$ and $\pi(r)$ satisfy the Euler-Lagrange equations, and the quark wave function satisfies the Dirac eigenvalue equation. Substituting Eq. in Euler-Lagrange equation, we get

$$
\begin{gathered}
\square \sigma^{\prime}=g \bar{\Psi} \Psi-\lambda_{1}^{2}\left(f_{\pi}-\sigma^{\prime}\right)\left(\left(\sigma^{\prime}-f_{\pi}\right)^{2}+\pi^{2}-\nu_{1}^{2}\right)- \\
2 \lambda_{2}^{2}\left(f_{\pi}-\sigma^{\prime}\right)\left(\left(\sigma^{\prime}-f_{\pi}\right)^{2}+\pi^{2}\right)\left(\left(\left(\sigma^{\prime}-f_{\pi}\right)^{2}+\pi^{2}\right)^{2}-\nu_{2}^{2}\right)-m_{\pi}^{2} f_{\pi}, \\
\left.\square \pi=i g \bar{\Psi} \gamma_{5} \cdot \tau \Psi-\lambda_{1}^{2}\left(\left(\sigma^{\prime}-f_{\pi}\right)^{2}+\pi^{2}-\nu_{1}^{2}\right)\right) \pi- \\
2 \lambda_{2}^{2} \pi\left(\left(\sigma^{\prime}-f_{\pi}\right)^{2}+\pi^{2}\right)\left(\left(\left(\sigma^{\prime}-f_{\pi}\right)^{2}+\pi^{2}\right)^{2}-\nu_{2}^{2}\right)
\end{gathered}
$$

where $\tau$ refers to Pauli isospin matrices, $\gamma_{5}=\left(\begin{array}{ll}0 & 1 \\ 1 & 0\end{array}\right)$. Including the color degree of freedom, one has $g \bar{\Psi} \Psi \rightarrow N_{c} g \bar{\Psi} \Psi$ where $N_{c}=3$ colors. Thus

$$
\Psi(r)=\frac{1}{\sqrt{4 \pi}}\left[\begin{array}{c}
u(r) \\
i w(r)
\end{array}\right] \quad \text { and } \quad \bar{\Psi}(r)=\frac{1}{\sqrt{4 \pi}}[u(r) i w(r)]
$$

then

$$
\begin{aligned}
& \rho_{s}=N_{c} \bar{\Psi} \Psi=\frac{3 g}{4 \pi}\left(u^{2}-w^{2}\right), \\
& \rho_{p}=i N_{c} \bar{\Psi} \gamma_{5} \tau \Psi=\frac{3 g}{2 \pi}(u w), \\
& \rho_{v}=\frac{3 g}{4 \pi}\left(u^{2}+w^{2}\right)
\end{aligned}
$$

where $\rho_{s}, \rho_{p}$ and $\rho_{v}$ are sigma, pion and vector densities, respectively. These equations are subject to the boundary conditions as follows,

$$
\sigma(r) \sim-f_{\pi}, \quad \pi(r) \sim 0 \quad \text { at } r \rightarrow \infty .
$$


By using hedgehog ansatz [12], where

$$
\pi(r)=\pi(r) \hat{\mathbf{r}}
$$

The chiral Dirac equation for the quarks is [12]

$$
\frac{d u}{d r}=-P(r) u+\left(W+m_{q}-S(r)\right) w
$$

where the scalar potential $S(r)=g\left\langle\sigma^{\prime}\right\rangle$, the pseudoscalar potential $P(r)=\langle\pi \cdot \hat{\mathbf{r}}\rangle$, and $W$ is the eigenvalue of the quarks spinor $\Psi$

$$
\frac{d w}{d r}=-\left(W-m_{q}+S(r)\right) u-\left(\frac{2}{r}-P(r)\right) w .
$$

\section{NUMERICAL CALCULATIONS AND DISCUSSIONS}

\section{A. The scalar field $\sigma^{\prime}$}

To solve Eq. (13), we integrate a suitable Green's function over the source fields as in Refs. [12,13]. Thus

$$
\begin{array}{r}
\sigma^{\prime}(\mathbf{r})=\int d^{3} \mathbf{r}^{\prime} D_{\sigma}(\mathbf{r}-\grave{\mathbf{r}})\left[g \rho_{s}(\grave{\mathbf{r}})-\lambda_{1}^{2}\left(f_{\pi}-\sigma^{\prime}\right)\left(\left(\sigma^{\prime}-f_{\pi}\right)^{2}+\pi^{2}-\nu_{1}^{2}\right)-\right. \\
\left.2 \lambda_{2}^{2}\left(f_{\pi}-\sigma^{\prime}\right)\left(\left(\sigma^{\prime}-f_{\pi}\right)^{2}+\pi^{2}\right)\left(\left(\left(\sigma^{\prime}-f_{\pi}\right)^{2}+\pi^{2}\right)^{2}-\nu_{2}^{2}\right)-m_{\pi}^{2} f_{\pi}\right],
\end{array}
$$

where

$$
D_{\sigma}(\mathbf{r}-\grave{\mathbf{r}})=\frac{1}{4 \pi|\mathbf{r}-\grave{\mathbf{r}}|} \exp \left(-m_{\sigma}|\mathbf{r}-\grave{\mathbf{r}}|\right),
$$

the scalar field is spherical in this model so we only need the $l=0$ term

$$
D_{\sigma}(\mathbf{r}-\grave{\mathbf{r}})=\frac{1}{4 \pi} \sinh \left(m_{\sigma} r_{<}\right) \frac{\exp \left(-m_{\sigma} r_{>}\right)}{r_{>}}
$$

therefore

$$
\begin{aligned}
\sigma^{\prime}(\mathbf{r}) & =m_{\sigma} \int_{0}^{\infty} r^{\prime 2} d r^{\prime}\left(\frac{\sinh \left(m_{\sigma} r_{>}\right) \exp \left(-m_{\sigma} r_{>}\right)}{m_{\sigma} r_{>}}\right)\left[g \rho_{s}(\grave{\mathbf{r}})-\right. \\
& \lambda_{1}^{2}\left(f_{\pi}-\sigma^{\prime}\right)\left(\left(\sigma^{\prime}-f_{\pi}\right)^{2}+\pi^{2}-\nu_{1}^{2}\right)-2 \lambda_{2}^{2}\left(f_{\pi}-\sigma^{\prime}\right)\left(\left(\sigma^{\prime}-f_{\pi}\right)^{2}+\pi^{2}\right) \times \\
& \left.\times\left(\left(\left(\sigma^{\prime}-f_{\pi}\right)^{2}+\pi^{2}\right)^{2}-\nu_{2}^{2}\right)-m_{\pi}^{2} f_{\pi}\right] .
\end{aligned}
$$

Note that this form is implicit in the solution of $\sigma^{\prime}$ involves integrals over the unknown $\sigma^{\prime}$ itself. We will solve this implicit integral equation by iterating to self-consistency. 


\section{B. The pion field $\pi$}

To solve Eq. (14), we integrate a suitable Green's function over the source fields. We use the $l=1$ component of the pion Green's function. Thus

$$
\begin{gathered}
\pi(r)=m_{\pi} \int_{0}^{\infty} r^{\prime 2} d r^{\prime} \frac{\left[-\sinh \left(m_{\pi} r_{<}\right)+m_{\pi} r_{<} \cosh \left(m_{\pi} r_{<}\right)\right]}{\left(m_{\pi} r_{>}\right)^{2}} \times \\
{\left[\left(1+\frac{1}{m_{\pi} r_{>}}\right) \frac{\exp \left(-m_{\pi} r_{>}\right)}{m_{\pi} r_{>}}\right)\left(g \rho_{p}-\lambda_{1}^{2}\left(\left(\sigma^{\prime}-f_{\pi}\right)^{2}+\pi^{2}-\nu_{1}^{2}\right)\right) \pi-} \\
\left.2 \lambda_{2}^{2} \pi\left(\left(\sigma^{\prime}-f_{\pi}\right)^{2}+\pi^{2}\right)\left(\left(\left(\sigma^{\prime}-f_{\pi}\right)^{2}+\pi^{2}\right)^{2}-\nu_{2}^{2}\right)\right] .
\end{gathered}
$$

We have solved Dirac Eqs. (21), (22) using fourth-order Rung Kutta method. Due to the implicit nonlinearly of these Eqs. (13), (14) it is necessary to iterate the solution until selfconsistency is achieved. To start this iteration process, we could use the chiral circle form for the meson fields $[12,13]$ :

$$
S(r)=m_{q}(1-\cos \theta), P(r)=-m_{q} \sin \theta,
$$

where $\theta=\tanh r$. 


\section{The Properties of the Nucleon}

The proton and neutron magnetic moments are given by [3]

$$
\mu_{p, n}=<P \uparrow\left|\int \frac{1}{2} \mathbf{r} \times \mathbf{j}_{\varepsilon M}(\mathbf{r}) d^{3} \mathbf{r}\right| P \uparrow>
$$

where, the electromagnetic current is

$$
j_{\epsilon M}(\mathbf{r})=\bar{\Psi}(\mathbf{r}) \gamma\left(\frac{1}{6}+\frac{\tau_{3}}{2}\right) \Psi(\mathbf{r})-\varepsilon_{\alpha \beta_{3}} \pi_{\alpha}(\mathbf{r}) \nabla \pi_{\beta}(\mathbf{r}),
$$

such that

$$
\begin{gathered}
\left(\mathbf{j}_{\epsilon M}(\mathbf{r})\right)_{\text {nucleon }}=\bar{\Psi}(\mathbf{r}) \gamma\left(\frac{1}{6}+\frac{\tau_{3}}{2}\right) \Psi(\mathbf{r}), \\
\left(\mathbf{j}_{\epsilon M}(\mathbf{r})\right)_{\text {meson }}=-\epsilon_{\alpha \beta 3} \pi_{\alpha}(\mathbf{r}) \nabla \pi_{\beta}(\mathbf{r}) .
\end{gathered}
$$

The nucleon axial-vector coupling constant is found from

$$
\frac{1}{2} g_{A}(0)=\left\langle P \uparrow\left|\int d^{3} r A_{3}^{z}(\mathbf{r})\right| P \uparrow\right\rangle,
$$

where the z-component of the axial vector current is given by

$$
A_{3}^{z}(\mathbf{r})=\bar{\Psi}(\mathbf{r}) \frac{1}{2} \gamma_{5} \gamma^{3} \tau_{3} \Psi(\mathbf{r})-\sigma(\mathbf{r}) \frac{\partial}{\partial z} \pi_{3}(\mathbf{r})+\pi_{3}(\mathbf{r}) \frac{\partial}{\partial z} \sigma(\mathbf{r}) .
$$

The pion-nucleus $\sigma$ commutator is defined

$$
\sigma(\pi N)=\left\langle P \uparrow\left|\int \sigma^{\prime}(\mathbf{r}) d^{3} r\right| P \uparrow\right\rangle
$$

In calculation of $\sigma(\pi N)$, we replace $\sigma^{\prime}(\mathbf{r})$ by $\frac{j_{\sigma}(\mathbf{r})}{m_{\sigma}^{2}}$ where $j_{\sigma}(\mathbf{r})$ is the source current defined by

$$
\left(\square+m_{\sigma}^{2}\right) \sigma^{\prime}=j_{\sigma}(\mathbf{r})
$$

The hedgehog mass is calculated in details in Refs. $[12,13]$ 


\section{Discussion of the Results}

The set of equations (13-22) are numerically solved by the iteration method as Refs. [12-14] for different values of the sigma and quark masses. The dependence of the nucleon properties on the sigma and the quark masses are listed in the tables (1), (2), (3), and (4). In Table (1), we note that the hedgehog mass, the magnetic moments of the proton and neutron, and the sigma commutator increase by increasing sigma mass. We obtain a good value of the hedgehog mass equals to $1090 \mathrm{MeV}$ which closed to experimental data 1086 $\mathrm{MeV}$. In Table (2), we examine the effect of quark mass on the nucleon properties. We note that the hedgehog mass decreases with increasing quark mass. This interpreted that an increase in the quark mass leads to increase in the coupling constant $\left(g=\frac{m_{q}}{f_{\pi}}\right)$. Therefore, the coupling between meson and the quark more tight, leading the decrease in the hedgehog mass as in Refs. $[3,12,13]$. Also, we note that the magnetic moments of proton and neutron increase by increasing quark mass. A similar effect occurred respect to sigma commutator $\sigma(\pi N)$. In comparison between the results in the tables 1 and 2 . We note that quark mass is more affected on nucleon properties that the strong change of sigma mass leads to the change of nucleon properties as in the table 1. In Table (3), we compare between the original quark model and the higher-order quark model. We fixed all parameters in the two models to show the effect of the higher-order mesonic interactions on the nucleon properties. We note that the dynamic of kinetic energy of quark increases by increasing mesonic contributions in the original quark model. In addition, the meson-quark interaction energy decreases by increasing higher-order interactions. We note that meson-meson interaction decreases by increasing mesonic contributions in the original sigma model. We obtain the excellent value of hedgehog mass $M_{H} \cong 1090 \mathrm{MeV}$ while we obtain $M_{H} \cong 1068 \mathrm{MeV}$ in the original sigma model at the same free parameters. Therefore, an increase of the mesonic interactions improved the hedgehog mass which closed to experimental data $\left(M_{H} \cong 1086 \mathrm{MeV}\right)$. The magnetic moments of proton and neutron are improved in comparison with the original model. Sigma commutator $\sigma(\pi N)$ is one of problems in the original sigma model that is a largest value in comparison with data. By increasing mesonic contributions in the original sigma model. This value reduced from $126 \mathrm{MeV}$ to $78 \mathrm{MeV}$. Therefore, the value improved about $38 \%$ and it is acceptable agreement with experimental data. The quantity $g_{A}(0)$ is improve in comparsion with the original model but still a large value in comparing with 
experimental data (1.25). Since the $g_{A}(0)$ depends on the meson fields only not on the coupling of higher-order term in the extended sigma model. Therefore, we need to add a vector meson to our model to improve this quantity, which will be a future paper.

Table (1). Values of magnetic moments of proton and neutron, the hedgehog mass $M_{B}$, and $\sigma(\pi N)$ for $m_{\pi}=139.6 \mathrm{MeV}, m_{q}=500 \mathrm{MeV}, f_{\pi}=92.4 \mathrm{MeV}$. All quantities in $\mathrm{MeV}$.

\begin{tabular}{|l|l|l|l|l|}
\hline$m_{\sigma}(\mathrm{MeV})$ & 600 & 700 & 800 & 900 \\
\hline Hedgehog mass $M_{B}$ & 1090.92 & 1108.98 & 1125.54 & 1139.27 \\
\hline Total moment proton $\mu_{p}(N)$ & 2.8456 & 2.8641 & 2.8643 & 2.8646 \\
\hline Total moment neutron $\mu_{n}(N)$ & -2.2076 & -2.2374 & -2.2494 & -2.259 \\
\hline$\sigma(\pi N)$ & 77.025 & 78.158 & 78.440 & 78.770 \\
\hline
\end{tabular}

Table (2). Values of magnetic moments of proton and neutron, the hedgehog mass $M_{B}$, and $\sigma(\pi N)$ for $m_{\pi}=139.6 \mathrm{MeV}, m_{\sigma}=600 \mathrm{MeV}, f_{\pi}=92.4 \mathrm{MeV}$. All quantities in MeV.

\begin{tabular}{|l|l|l|l|l|l|l|}
\hline$m_{q}(\mathrm{MeV})$ & 400 & 420 & 440 & 460 & 480 & 500 \\
\hline Hedgehog mass $M_{B}$ & 1230 & 1210 & 1185 & 1157 & 1124 & 1089 \\
\hline Total moment proton $\mu_{p}(N)$ & 2.574 & 2.653 & 2.719 & 2.775 & 2.823 & 2.845 \\
\hline Total moment neutron $\mu_{n}(N)$ & -1.899 & -1.985 & -2.05 & -2.121 & -2.175 & -2.207 \\
\hline$\sigma(\pi N)$ & 49.19 & 57.57 & 64.28 & 69.79 & 74.32 & 77.02 \\
\hline
\end{tabular}

Table(3). Details of energy calculations of the hedgehog mass, the magnetic moments of proton and neutron, and the sigma commutator $\sigma(\pi N)$ for $m_{q}=500 \mathrm{MeV}, m_{\pi}=139.6$ $\mathrm{MeV}, m_{\sigma}=600 \mathrm{MeV}$, and $f_{\pi}=92.4 \mathrm{MeV}$. All quantities in $\mathrm{MeV}$. 


\begin{tabular}{|l|l|l|}
\hline Quantity & Original Sigma Model & Higher-order Sigma Model \\
\hline Quark kinetic energy & 1166.38 & 1171.068 \\
\hline Sigma kinetic energy & 353.15 & 375.038 \\
\hline Pion kinetic energy & 461.85 & 451.827 \\
\hline Sigma interaction energy & -165.84 & -165.975 \\
\hline Pion interaction energy & -860.87 & -854.098 \\
\hline Meson interaction energy & 114.0 & 113.069 \\
\hline Hedgehog mass baryon & 1068.67 & 1090.92 \\
\hline Total moment of proton $\mu_{p}$ & 2.89 & 2.84 \\
\hline Total moment of neutron $\mu_{n}$ & -2.24 & 2.20 \\
\hline$g_{A}(0)$ & 1.80 & 1.78 \\
\hline$\sigma(\pi N)$ & 126.99 & 77 \\
\hline
\end{tabular}

\section{COMPARISON WITH OTHER MODELS}

It is interesting to compare the nucleon properties in the present approach with the previous works and other models. The higher-order mesonic potential was suggested in Refs. [12-14]. In Ref. [12], the sigma commutator $\sigma(\pi N)$ is not calculated in this work. It is an essential property of nucleon properties. In addition, the mesonic potential has a weakness point at $m_{\pi}=0$ so the model did not satisfy the chiral limit case. We note that the hedgehog mass improved in comparison with result of Ref. [12 ]. In Ref. [13], the authors suggested another form of mesonic potential to avoid the difficulty which came from $m_{\pi}=0$. We have two advantages in comparison with Ref. [13]. The first, our results in the present work are improved, in particular the hedgehog mass and the $\sigma(\pi N)$. The second, the mesonic potential in Eq. 7, has the similar form when the coupling constant of higher-order $\lambda_{2}^{2}$ is vanished as in Eq. 2. This advantage is not found in Ref. [13]. In Ref. [14], the author studied the effect of large pion masses on the magnetic moments of proton and neutron only.

It is important to compare present model with other models such as the perturbative chiral quark Model $[6,7]$ and the extended Skyrme model [15]. The perturbative chiral quark model is an effective model of baryons based on chiral symmetry. The baryon is described as a state of three localized relativistic quarks supplemented by a pseudoscalar 
meson cloud as dictated by chiral symmetry requirements. In this model, the effect of the meson cloud is evaluated perturbatively in a systematic fashion. The model has been successfully applied to the nucleon properties (see Table 4). We obtain reasonable results in comparison with this model for the $\sigma(\pi N)$ which backs to perturbative chiral quark model based on non-linear $\sigma$ - model Lagrangian. In particular, nucleon magnetic moments are improved in comparison with this model. Moreover, Hedgehog mass $M_{B}$ is not calculated in this model. The original Skyrme model [16] consists of the non-linear sigma term and the fourth-order derivative term, which guarantees the stabilization of the soliton so that the degree of freedom of the sigma field may be replaced by a variable chiral radius, which becomes the new dynamical degree of freedom and plays an important role in the modified Skyrmion Lagrangian density [15], leading to a better description of nucleon properties. In comparison with the extended Skyrme model [15], the results obtained for the hedgehog mass have been improved and the other properties are in agreement with this model (see Table 4).

Table (4). Values of the observables calculated from the extended linear sigma model $[12,13]$, the perturbative chiral quark model $[6,7]$, and the extended Skyrme model [15] in comparison with the present work.

\begin{tabular}{|l|l|l|l|l|l|l|}
\hline Quantity & Present work & {$[13]$} & {$[6,7]$} & {$[12]$} & {$[15]$} & Expt. \\
\hline Hedgehog mass $M_{B}$ & 1090 & 1200 & - & 1081 & 1157 & 1086 \\
\hline$\mu_{p}(N)$ & 2.84 & 2.76 & $2.62 \pm 0.02$ & 2.768 & 2.77 & 2.79 \\
\hline$\mu_{n}(N)$ & -2.20 & -1.91 & $-2.02 \pm 0.02$ & -1.909 & -2.11 & -1.91 \\
\hline$\sigma(\pi N)$ & 77 & 88 & 54.7 & - & 70 & $50 \pm 20$ \\
\hline
\end{tabular}

\section{CONCLUSION}

The present calculations have shown the importance of mesonic corrections of higherorder than that normally used in most soliton models. The obtained results are improved in comparison with previous calculations. In addition, we avoid the difficulty that found in the previous works. The advantage of the present work that hedgehog mass is corrected and closed with data. The magnetic moments of proton and neutron and sigma commutator $\sigma(\pi N)$ are improved in comparison with other models. 


\section{REFERENCES}

1. S. Gasiorowicz and D. A. Geffen, Rev. Mod. Phys. 41, 531 (1969).

2. M. Gell-Mann, M. Levy, Nuovo Cimento 16, 705 (1960).

3. M. Birse and M. Banerjee, Phys. Rev. D 31, 118 (1985).

4. W. Broniowski and M. K. Banerjee, Phys. Lett. B 158, 335 (1985).

5. M. Birse, Phys. Rev. D 33, 1934 (1986).

6. V. E. Lyubovitskij, T. Gutsche and A. Faessler, Phys. Rev. C 64, 065203 (2001).

7. T. Inoue, V. E. Lyubovitskij, T. Gutsche, A. Faessler, Phys. Rev. C 69, 035207 (2004).

8. D. Horvat, D. Horvatic, B. Podobnik and D. Tadic, FIZIKA B 9, 181 (2000).

9. W. Broniowski and B. Golli, Nucl. Phys. A 714, 575 (2003).

10. M. Abu-Shady, Acta Phys. Polo. B 40, 8 (2009).

11. M. Abu-Shady, Int. J. Theor. Phys. 48, 1110 (2009).

12. M. Rashdan, M. Abu-Shady, and T.S.T Ali, Inter. J. Mod. Phys. A 22, 2673 (2007)

13. M. Rashdan, M. Abu-shady, and T.S.T. Ali, Int. J. Mod. Phys. E 15, 143 ( 2006).

14. M. Abu-Shady, Phys. Atom. Nucl. 73, 978 (2010).

15. F. L. Braghin and I. P. Cavalcante, Phys. Rev. C 67, 065207 (2003).

16. T. H. R. Skyrme, Proc. R. Soc. London, Ser. A 260, 127 (1961). 